\title{
PRODUÇÃO DO CUIDADO NA REDE DE ATENÇÃO AO CÂNCER DE MAMA: REVISÃO INTEGRATIVA
}

\author{
PRODUCTION OF CARE IN THE BREAST CANCER CARE NETWORK:
} INTEGRATIVE REVIEW

PRODUCCIÓN DEL CUIDAdO EN LA RED DE ATENCIÓN AL CÁNCER DE MAMA: REVISIÓN INTEGRADORA

Francisca Alanny Rocha Aguiar 1

Thaianny Cordeiro de Sousa ${ }^{2}$

July Grassiely de Oliveira Branco 3

Francisca Bertilia Chaves Costa ${ }^{4}$

Amelia Romana Almeida Torres ${ }^{5}$

Lidyane Parente Arruda 6

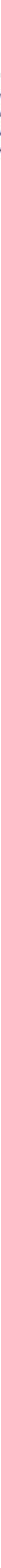

84 - SANARE, Sobral - v.17, n.01,p.84-92, Jan./Jun. - 2018 


\section{ABSTRACT}

Breast cancer has been chosen as a directive condition for this study because it is one of the most prevalent health issues in Brazil. From this perspective, interprofessional collaboration is a measure to be considered in order to ensure good-quality care. The aim was analyzing how production of care occurs in the breast cancer care network. This is an integrative review with search conducted in October 2016, on the Virtual Health Library (VHL), using the databases SCIELO, LILACS, and Coleciona SUS. The descriptors 'breast neoplasms,' 'cooperative behavior,' 'health services,' and 'Unified Health System' were cross-referenced. In view of the findings, the user's itinerary in the health system was identified, since her first appointment in the Primary Health Care (PHC) until the diagnosis was established. The difficulties of this system for effecting this line of care in the health service showed that lengthy waiting times can have serious consequences. It is worth emphasizing the importance of creating devices that increase interprofessional collaboration and the line of care in the breast cancer care network, enabling the provision of comprehensive and problem-solving actions.

\section{RESUMEN}

El cáncer de mama ha sido elegido como una condición directiva para este estudio porque es uno de los problemas de salud más prevalentes en Brasil. Desde esta perspectiva, la colaboración interprofesional es una medida a considerar para garantizar una atención de buena calidad. Se objetivó analizar cómo ocurre la producción del cuidado en la red de atención al cáncer de mama. Esta es una revisión integradora con búsqueda realizada en octubre de 2016, en la Biblioteca Virtual en Salud (BVS), utilizando las bases de datos SciELO, LILACS y Coleciona SUS. Se han cruzado los descriptores "neoplasias de la mama", "conducta cooperativa", "servicios de salud" y "Sistema Único de Salud". En vista de los hallazgos, se identificó el itinerario de la usuaria en el sistema de salud, desde su primera consulta en la Atención Primaria de Salud (APS) hasta que se establezca el diagnóstico. Las dificultades de este sistema para llevar a cabo esta linea de cuidado en el servicio de salud mostraron que los largos tiempos de espera pueden tener graves consecuencias. Vale la pena enfatizar la importancia de crear dispositivos que aumenten la colaboración interprofesional y la línea de cuidado en la red de atención al cáncer de mama, permitiendo la provisión de acciones integrales y resolutorias.

\section{INTRODUÇÃO}

A relação do homem ou das populações com sua história, seus padrões de desenvolvimento, suas interações com o mundo e com o ambiente sociocultural, suas necessidades, direitos e condições de vida reflete uma transição demográfica acelerada que acumula doenças infecciosas e parasitárias, problemas de saúde materno-infantis, causas externas e condições crônicas. Essa situação requer respostas sociais deliberadas às demandas de saúde da população $0^{1,2}$.

Observa-se a necessidade de cuidado longitudinal a ser prestado nas Redes de Atenção à Saúde (RAS), as quais atuam na prestação de serviços com diferentes densidades tecnológicas ao longo do sistema de saúde. Para garantir o cuidado integral, cada nível de atenção assume um papel específico com vistas à resolução dos problemas de saúde da população ${ }^{3}$.

Nesse contexto, manifestam-se especificidades nos campos de trabalho no setor saúde, revelando a urgência de uma interlocução entre os atores envolvidos nesses espaços de atenção que sejam capazes de desenvolver uma lógica de atendimento que se adapte à nova realidade. Essa lógica renovada cede à hegemonia da atenção aos eventos agudos para adoção de uma abordagem contínua que integre as dimensões biopsicossociais.

Destarte, o uso de práticas colaborativas pode assumir um papel importante no enfrentamento de muitos desafios do sistema de saúde; um deles seria a inclusão da rede de atenção à pessoa com condição crônica nas ações de prevenção e controle do câncer ${ }^{4,5}$. No Brasil, tem-se visto a ascensão das Doenças Crônicas Não Transmissíveis (DCNT), consideradas sério problema de saúde pública, que demandam ações de maior abrangência do sistema de saúde, além de maior promoção da saúde e prevenção de doenças, com vistas a qualificar a atenção às pessoas com doenças crônicas ${ }^{5}$.

Dentre as DCNT se destaca o câncer, definido como um conjunto de mais de 100 doenças que tem em comum o crescimento descontrolado de células anormais e sua disseminação, resultando em alterações morfológicas distintas e em anomalias nos padrões histológicos, podendo espalhar-se por diversas regiões do corpo $0^{6,7}$. 
Em 2011 se verificou aumento da incidência do câncer de mama na população brasileira, que ocupa o segundo lugar em número de mulheres afetadas no mundo, correspondendo a $25 \%$ de todos os casos de câncer diagnosticados em 2012 e apresentando a estimativa de 1,67 milhão de casos novos nesse ano. Tal agravo, dentre os cânceres, estabeleceu-se como o mais comum em mulheres, classificado como a quinta causa de morte mais frequente por câncer (522 mil óbitos) ${ }^{8}$.

No Brasil, de acordo com as estimativas para 2016/2017, esperava-se um registro próximo a 596 mil casos de câncer. Entre os homens são esperados 295.200 casos e entre as mulheres, 300.800 casos. 0 número de casos novos de câncer de mama estimado para 2016 foi de 57.960, excluindo os tumores de pele não melanoma. Trata-se do tipo mais incidente em mulheres de todas as regiões, exceto no Norte, onde o câncer do colo do útero ocupa a primeira posição $0^{9}$.

Existem diversas causas para o desenvolvimento do câncer de mama, internas ou externas ao organismo, que estão inter-relacionadas. As causas externas se relacionam ao meio ambiente e aos hábitos ou costumes próprios de um ambiente social e cultural. As causas internas são, na maioria das vezes, geneticamente determinadas e estão ligadas à capacidade do organismo se defender das agressões externas ${ }^{10}$.

Entretanto, considerando a gravidade desse tipo de câncer, o profissional da saúde tem assumido um papel cada vez mais decisivo e proativo diante das necessidades de cuidados básicos das mulheres em suas diferentes dimensões, ou seja, a implementação de estratégias eficazes que incentivem métodos preventivos e a conscientização da população para a adoção de práticas voltadas ao diagnóstico precoce, aumentando as chances de tratamento bemsucedido ${ }^{11}$.

Diante dessas considerações, o que se têm observado é que se um sistema de saúde funciona com base em uma lógica de rede, seus processos dependem especialmente de relações estabelecidas em parceria ${ }^{12}$. Assim, objetivou-se analisar na literatura científica como ocorre a produção do cuidado na rede de atenção ao câncer de mama.

\section{METODOLOGIA}

Trata-se de revisão integrativa, realizada por meio das seguintes etapas: a) identificação do tema; b) elaboração da questão norteadora; c) estabelecimento dos critérios de inclusão e exclusão de estudos; d) identificação dos estudos pré-selecionados e selecionados; e) categorização dos estudos selecionados; f) análise e interpretação dos resultados; e g) apresentação da síntese da revisão $0^{13}$.

Para consolidar os objetivos propostos, elaborouse a seguinte questão norteadora:

- Como se dá a produção do cuidado na rede de atenção ao câncer de mama?

Busca-se, a partir de busca na literatura, desvelar o trabalho em equipe $e$ as interfaces para 0 acompanhamento em rede de atenção para pacientes com câncer de mama.

0 cruzamento dos descritores ocorreu na Biblioteca Virtual em Saúde (BVS), utilizando as bases de dados SciELO, Lilacs e Coleciona SUS. As buscas foram realizadas em outubro de 2016 e tiveram a finalidade de identificar estudos publicados no período de 2012 a 2016.

Para a busca dos artigos foram adotados os seguintes descritores em ciências da saúde (DeCS): "neoplasias de mama", "comportamento cooperativo", "serviços de saúde" e "Sistema Único de Saúde" mediados pelo operador booleano "AND".

Os critérios de inclusão adotados foram: a) artigos de livre acesso; b) publicações em português; e c) artigos que apresentavam referências a cuidado em saúde, estratégias e/ou manejo de pacientes com câncer de mama, acesso/barreiras de acesso a serviços e conduta profissional (inclusive colaboração interprofissional e trabalho em equipe).

Já os critérios de exclusão foram: a) artigos com duplicidade de inclusão nas bases de dados; e b) artigos sem elementos relevantes quanto ao escopo da pesquisa.

Após leitura integral de cada artigo identificado, constatou-se que 9 publicações científicas deveriam ser selecionadas como fontes de dados para esta pesquisa (Figura 1).

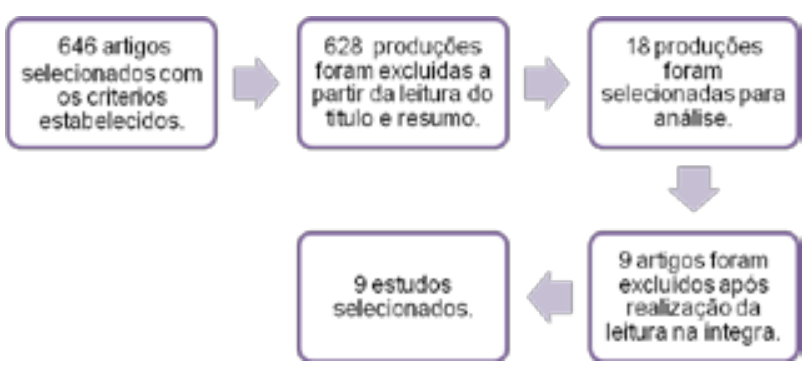

Figura 1. Seleção de artigos nas bases da Biblioteca Virtual em Saúde.

Fonte: Elaborada pelas autoras. 
Os dados foram obtidos mediante aplicação de um instrumento de coleta adaptado ${ }^{13}$ para análise de 4 variáveis: a) título do artigo; b) tipo de estudo; e c) base de dados. Tal análise gerou 2 categorias: a) Linha de cuidado ao câncer de mama na rede de atenção à saúde; e b) Desafios e potencialidades para a efetivação da linha de cuidado na atenção ao câncer de mama.

\section{RESULTADOS E DISCUSSÃO}

0 Quadro 1 caracteriza os artigos selecionados com base nas variáveis de análise.

Quadro 1 - Caracterização dos artigos selecionados.

\begin{tabular}{|c|c|c|}
\hline Título & Tipo de estudo & Base de dados \\
\hline $\begin{array}{l}\text { Assistência em mastologia em uma unidade de referência do } \\
\text { Sistema Único de Saúde no Ceará, Brasil }{ }^{14}\end{array}$ & $\begin{array}{l}\text { Estudo transversal e } \\
\text { quantitativo }\end{array}$ & Coleciona SUS \\
\hline $\begin{array}{l}\text { Atraso no diagnóstico e tratamento do câncer de mama e } \\
\text { estratégias para sua redução }{ }^{15}\end{array}$ & Revisão bibliográfica & Lilacs \\
\hline $\begin{array}{l}\text { Barreiras no rastreamento do câncer de mama e o papel da } \\
\text { enfermagem: revisão integrativa }{ }^{16}\end{array}$ & Revisão bibliográfica & SCIELO \\
\hline $\begin{array}{l}0 \text { retrato das políticas públicas no tratamento do câncer de } \\
\text { mama no Brasil }{ }^{17}\end{array}$ & Revisão bibliográfica & Lilacs \\
\hline $\begin{array}{l}\text { Conduta de profissionais durante a consulta de rastreio do } \\
\text { câncer de mama e útero }{ }^{18}\end{array}$ & Estudo descritivo & Lilacs \\
\hline $\begin{array}{l}\text { Acesso à detecção precoce do câncer de mama no Sistema } \\
\text { Único de Saúde: uma análise a partir dos dados do Sistema de } \\
\text { Informação em Saúde }{ }^{19}\end{array}$ & Estudo descritivo & SCIELO \\
\hline $\begin{array}{l}\text { Aspectos relacionados ao atraso no diagnóstico e tratamento } \\
\text { do câncer de mama em uma unidade hospitalar de Pernambuco }{ }^{20}\end{array}$ & $\begin{array}{l}\text { Estudo epidemiológico } \\
\text { observacional }\end{array}$ & Coleciona SUS \\
\hline $\begin{array}{l}\text { Os caminhos de cuidado das mulheres com diagnóstico de } \\
\text { câncer de mama }{ }^{21}\end{array}$ & Estudo descritivo & SCIELO \\
\hline $\begin{array}{l}\text { Demora no diagnóstico de câncer de mama de mulheres } \\
\text { atendidas no sistema público de saúde }{ }^{22}\end{array}$ & Estudo descritivo & SCIELO \\
\hline
\end{tabular}

Fonte: Elaborado pelas autoras.

Em relação à abordagem metodológica, prevalecem os estudos transversais descritivos (4), seguidos pelas revisões bibliográficas (3) e pelos estudos epidemiológicos observacionais (1) e transversais quantitativos (1). Com base na análise das publicações, a discussão englobou as categorias descritas a seguir.

\section{Linha de cuidado ao câncer de mama na rede de atenção à saúde}

0 aumento da incidência de câncer de mama no Brasil tem sido acompanhado pelo aumento da mortalidade - o que pode ser atribuído ao diagnóstico da doença em estágios avançados, sugerindo a dificuldade de acesso aos serviços de saúde ${ }^{22}$.

A detecção precoce abrange duas estratégias: a) o diagnóstico precoce; e b) o rastreamento. 0 diagnóstico precoce consiste na abordagem de pessoas com sinais e/ou sintomas da doença. Já o rastreamento é a aplicação de teste ou exame em uma população assintomática (aparentemente saudável), com a finalidade de identificar lesões sugestivas de câncer ${ }^{23}$.

No que tange à assistência, as ações de rastreamento para o diagnóstico precoce fazem parte das atribuições dos serviços de Atenção Primária à Saúde (APS), ao passo que a investigação para a efetivação do diagnóstico e o tratamento fazem parte do conjunto de ações dos serviços especializados da atenção secundária e terciária, respectivamente ${ }^{18,22}$.

A concentração das ações de rastreamento na APS ocorre não apenas por ser a principal porta de entrada no Sistema Único de Saúde (SUS), mas, fundamentalmente, em função da natureza da densidade tecnológica prevista para esse nível de atenção e da proximidade entre a equipe e os usuários dos serviços de saúde, estreitada à medida que o 
vínculo se estabelece no cotidiano da assistência ${ }^{22}$.

$\mathrm{Na}$ unidade de saúde, os profissionais que identificam uma paciente assintomática na faixa etária recomendada pelo Ministério da Saúde devem solicitar a "mamografia de rastreamento" por meio de formulário padronizado e providenciar seu encaminhamento. No caso das mulheres sintomáticas em qualquer faixa etária, o pedido deve ser feito com indicação clínica de "mamografia diagnóstica" 24 . 0 Quadro 2 ilustra o itinerário percorrido pela usuária no sistema de saúde.

Quadro 2 - Itinerário da usuária para realização de mamografia, biopsia e cirurgia para tratamento do câncer de mama.

\begin{tabular}{|c|c|}
\hline Nível de atenção & Percurso da usuária \\
\hline Unidade de saúde (atenção primária) & Recebimento de solicitação da mamografia. \\
\hline $\begin{array}{l}\text { Serviço de mamografia (atenção } \\
\text { secundária) }\end{array}$ & Realização da mamografia e recebimento do resultado. \\
\hline Unidade de saúde (atenção primária) & $\begin{array}{l}\text { Retorno ao médico solicitante com o laudo da mamografia. Se o } \\
\text { laudo da mamografia se enquadrar nas categorias BI-RADS } 4 \text { ou } 5 \text {, há } \\
\text { encaminhamento para unidade especializada. }\end{array}$ \\
\hline $\begin{array}{l}\text { Unidade especializada (atenção } \\
\text { secundária) }\end{array}$ & Recebimento da solicitação e realização da biopsia. \\
\hline Laboratório (atenção secundaria) & Emissão do resultado da biopsia. \\
\hline $\begin{array}{l}\text { Unidade especializada (atenção } \\
\text { secundaria) }\end{array}$ & $\begin{array}{l}\text { Se o laudo da biopsia indica doença benigna, há orientação médica } \\
\text { sobre a conduta terapêutica. Se o laudo da biopsia indica doença } \\
\text { maligna, há encaminhamento para cirurgia. }\end{array}$ \\
\hline Atenção terciária & Realização da cirurgia. Tratamento. \\
\hline
\end{tabular}

Fonte: Adaptado de Azevedo e Silva, Bustamante-Teixeira, Aquino, Tomazelli, Santos (2014) ${ }^{19}$.

Como mostra o Quadro 2, após a consulta na unidade de APS, a paciente é encaminhada ao serviço de atenção secundária para realização da mamografia. 0 laudo de mamografia padronizado com base no sistema de classificação BI-RADS é entregue à usuária, que deve retornar ao profissional que solicitou o exame para análise do resultado e indicação da conduta adequada. Por sua vez, o encaminhamento para conclusão do diagnóstico se apoia na interpretação das categorias BI-RADS ${ }^{19}$. Quando há confirmação do diagnóstico, havendo indicação de tratamento cirúrgico, a usuária é encaminhada ao nível terciário de atenção (hospital especializado em cirurgia mamária) ${ }^{19}$.

A linha de cuidado baseada na colaboração interprofissional pressupõe ações integradas dos recursos disponíveis - serviços, equipes de saúde e comunidade organizada -, voltados a atender às demandas de saúde dos usuários, a reduzir a morbimortalidade e a melhorar a qualidade de vida dos portadores de doenças. Tudo isso requer ações coordenadas que envolvam estratégias de prevenção, detecção precoce, tratamento oportuno e cuidados paliativos, complementadas por ações que perpassam os campos da educação e comunicação em saúde, da vigilância do câncer e dos fatores de risco à saúde ${ }^{22}$.

A linha de cuidado consiste na imagem pensada para expressar os fluxos assistenciais demandados em situações de saúde/doença, com vistas a garantir o atendimento às necessidades de saúde. Aplicada ao câncer de mama, a linha de cuidado é o itinerário que a usuária percorre para garantir acesso à assistência integral de que necessita, inclusive as ações oferecidas nos três níveis de atenção e de assistência à saúde, além daquelas ofertadas pelas entidades comunitárias e sem fins lucrativos, integradas à rede para proporcionar suporte social ao paciente e à família 22 .

A efetividade do cuidado dispensado depende de pactuação do fluxo entre os serviços e de reorganização dos processos de trabalho. A quebra do fluxo de serviços ou a inexistência de articulação entre eles costuma dificultar o percurso das usuárias na rede de saúde e atrasar a concretização das ações, retardando o diagnóstico, o início do tratamento ou mesmo as ações de natureza social, fundamentais em situações de vulnerabilidade social.

0 tempo gasto pelas mulheres em cada etapa da assistência expõe gargalos importantes na agenda de consulta em serviços especializados da atenção secundária e na biopsia. Esse período temporal ao longo do itinerário pode levar a um prognóstico desfavorável 22 . 


\section{Desafios e potencialidades para a efetivação da linha de cuidado na atenção ao câncer de mama}

A demora para estabelecer o diagnóstico e iniciar o tratamento pode trazer graves consequências às usuárias com câncer de mama - associadas a menor taxa de sobrevida ${ }^{20,22}$. Trata-se do tempo transcorrido entre o primeiro contato com o serviço que gerou a solicitação de exame de imagem até a efetivação do diagnóstico, além do tempo parcial entre cada evento, como o exame de mamografia e/ou a ultrassonografia, a consulta médica especializada e a biopsia ${ }^{22}$.

Quanto à diferença entre a demora de atendimento às usuárias que residem na capital cearense e àquelas provenientes do interior do estado em um centro de referência, a mediana do tempo de espera por consulta especializada foi de 15 dias para as usuárias da capital e 30 dias para as usuárias do interior do estado. Diante da solicitação de biopsia, seu resultado, além da realização e do resultado de mamografia, não se identificou diferença em relação ao tempo de espera, com média de 30 a 60 dias. Quanto ao procedimento cirúrgico, a média do tempo de espera para usuárias da capital foi de 39 dias e de 63 dias para as usuárias do interior ${ }^{14}$.

0 acesso ao diagnóstico e ao tratamento varia de região para região do país, dependendo de variáveis geográficas e sociais relativas à distância entre a residência e os serviços de saúde, do nível de informação das mulheres e da disponibilidade de serviços. 0 mapa da mortalidade por câncer de mama indica a influência desses fatores, que é maior nas regiões onde a incidência da doença é elevada e os recursos médicos para diagnóstico precoce e tratamento adequado são de difícil acesso ${ }^{22}$. Há, ainda, imensas desigualdades na oferta de assistência especializada ${ }^{19}$.

A média de demora entre o atendimento no serviço de saúde e a confirmação do diagnóstico pode atingir 120 a 180 dias de espera, com chance do diagnóstico se concretizar em 60 dias. Já os encaminhamentos podem apresentar uma média superior a 30 dias $^{22}$.

Destaca-se que a Lei Federal nº.12.732/2012 garante a todo usuário com neoplasia o direito de submeter-se ao primeiro tratamento dentro do prazo de 60 dias (contados a partir do dia em que for estabelecido o diagnóstico $)^{24}$.

0 principal fator de demora relatado foi a espera por consulta médica especializada no nível secundário de atenção, visto que ações desenvolvidas no âmbito

\section{...os resultados reforçam as potencialidades da APS como porta de entrada no SUS...}

da detecção precoce e do diagnóstico apresentaram enorme avanço; mas essa espera no itinerário das usuárias na busca pelo diagnóstico demonstra a fragilidade na efetivação da linha de cuidados oncológicos ${ }^{22}$.

Outro fator que dificulta essa rede de cuidados decorre do limitado número de casos com diagnóstico precoce. 0 rastreamento mamográfico ainda constitui o melhor método de prevenção, com medidas de intervenção, detecção precoce na fase assintomática e diminuição da morbimortalidade decorrente do diagnóstico tardio. No entanto, há diversas barreiras relacionadas ao rastreamento mamográfico que podem advir do sistema de saúde, da educação e da usuária ${ }^{16}$.

Assim, os resultados reforçam as potencialidades da APS como porta de entrada no SUS, tendo como responsabilidades na atenção ao câncer de mama seu rastreamento e o fortalecimento do vínculo entre as usuárias e o serviço de saúde, contribuindo para a criação de uma linha de cuidado dentro da rede de atenção oncológica ${ }^{25}$. Delineia-se, ainda, a importância de articular as ações de educação em saúde como produtoras de um saber coletivo que estimula 0 indivíduo quanto à sua autonomia e emancipação para o autocuidado e o cuidado voltado à sua família e ao seu ambiente.

Um estudo realizado no norte do Ceará apontou que atividades grupais com mulheres mastectomizadas em processo de reabilitação com ações de educação em saúde propiciou que as informações referentes ao câncer de mama e seu acompanhamento fossem mais acessiveis, proporcionando melhoria da qualidade de vida dessas usuárias ${ }^{26}$.

As estratégias também incluem promover 0 diagnóstico e tratamento precoce, minimizando qualquer atraso no itinerário da usuária, além da capacitação dos profissionais da saúde, sendo estes da APS ou não, além dos agentes comunitários de saúde $(A C S)^{15}$.

Desse modo, destaca-se a importância dos profissionais da saúde no processo saúde-doença suas orientações e condutas influenciam 
significativamente 0 percurso dos portadores de doença. 0 enfermeiro, em especial, pode desenvolver ações para esclarecer dúvidas e proporcionar maior segurança aos usuários.

Considerando que a precocidade do diagnóstico e do tratamento reduz os impactos das doenças, a demora pode ser minimizada pela implementação efetiva de uma linha de cuidados na rede de serviços oncológicos, com pactuação dos fluxos entre os serviços para agilizar o acesso das usuárias à assistência integral ${ }^{22}$.

A implantação de um programa que auxilie ao longo do percurso, do diagnóstico ao tratamento, mostrou grande relevância na redução do tempo tanto para o diagnóstico como para o tratamento, auxiliando a transposição das barreiras de acesso aos serviços de saúde e aumentando a compreensão das usuárias sobre o tratamento e a importância da adesão para um bom prognóstico ${ }^{15}$.

A maior proporção da redução da mortalidade por câncer de mama foi atribuída ao diagnóstico precoce associado à melhoria da gestão da assistência às usuárias ${ }^{15}$.

Como modo de solucionar os problemas apresentados, bem como para avançar rumo às potencialidades, sugere-se: a) ampliação do rastreamento mamográfico anual em todo o Brasil para as faixas etárias preconizadas; b) capacitação dos serviços de rastreamento e diagnóstico por imagem; e c) criação de novos centros de referência para o paciente oncológico. Além disso, há necessidade de melhor alocação dos recursos públicos na promoção da saúde e na prevenção e no tratamento de doenças ${ }^{17}$.

\section{CONCLUSÃO}

Compreende-se a importância da melhoria de acesso aos serviços de saúde e da qualidade do atendimento, vislumbrando o relacionamento interpessoal e o cuidado integral ao ser humano pondo em prática os princípios de universalidade, integralidade e equidade preconizados pelo SUS.

Assim, a linha de cuidado demonstra uma possibilidade de garantir a integralidade relacionada à organização dos serviços de saúde, mostrando o caminho a ser percorrido nas redes de atenção ao câncer de mama - desde a Estratégia Saúde da Família (ESF) e sempre levando em conta a colaboração interprofissional como recurso de fortalecimento dessa linha de cuidado.

\section{... a linha de cuidado demonstra uma possibilidade de garantir a integralidade relacionada à organização dos serviços de saúde...}

Quanto ao tempo de demora, as usuárias acometidas por câncer de mama precisam esperar além do que é recomendado; com isso, constata-se a necessidade de implantação efetiva da linha de cuidado na rede de atenção oncológica, com pactuação entre os serviços para agilizar o acesso à assistência integral.

É evidente que as ações desenvolvidas no âmbito da detecção precoce e do diagnóstico do câncer de mama no Brasil obtiveram enormes avanços. As leis sancionadas com vistas ao tratamento também representam conquistas importantes, no entanto, a falta de estrutura e investimento no setor saúde são fatores limitantes para o cumprimento dessas normativas e para a prestação de um atendimento adequado às usuárias com esse tipo de neoplasia.

Pode-se afirmar que os objetivos propostos neste estudo foram alcançados, visto que foi possível, por meio da análise dos artigos, descrever o processo colaborativo na atenção do câncer de mama, apesar da carência de estudos que abordam essa temática.

Enfatizamos a importância da criação de dispositivos que ampliem a colaboração interprofissional nos serviços de saúde, possibilitando a prestação de cuidados integrais e resolutivos e diminuindo as dificuldades no itinerário desde 0 diagnóstico até o tratamento do câncer de mama.

Esperamos que este estudo constitua ferramenta de apoio para garantir uma melhor atenção à mulher com câncer de mama, mediante organização dos fluxos assistenciais e fortalecimento das ações na rede de atenção oncológica. Sabemos que os desafios são diversos, mas também se mostram estimulantes e promovem o compromisso e a determinação dos profissionais da saúde.

\section{CONTRIBUIÇÃO DAS AUTORAS}

Francisca Alanny Rocha Aguiar e Thaianny Cordeiro de Sousa contribuíram com o delineamento 
da pesquisa e a estruturação e redação do manuscrito. July Grassiely de Oliveira Branco e Francisca Bertilia Chaves Costa contribuíram com a redação do manuscrito. Amelia Romana Almeida Torres e Lidyane Parente Arruda contribuíram com a revisão crítica do manuscrito.

\section{REFERÊNCIAS}

1. Moretti AC, Teixeira FF, Suss FMB, Lawder Juliana AC, Lima LSM, Bueno RE, et al. Intersetorialidade nas ações de promoção de saúde realizadas pelas equipes de saúde bucal de Curitiba (PR). Ciênc Saúde Colet [serial on the internet]. 2010 [cited 2018 May 4];15(Suppl 1):1827-34. Available from: http://www.scielo.br/pdf/csc/v15s1/095.pdf

2. Mendes EV. As redes de atenção à saúde. Brasília (DF): Organização Pan-Americana da Saúde; 2011.

3. Brasil. Diretrizes para a organização das RAS no âmbito do SUS. Portaria n. 4.279, de 30/12/2010. Brasília (DF): Ministério da Saúde; 2010.

4. Organização Mundial da Saúde. Marco para ação em educação interprofissional e prática colaborativa. Redes de profissões de saúde. Enfermagem e obstetrícia. Recursos humanos para a saúde. Brasília (DF): Organização Mundial da Saúde; 2010.

5. Brasil. Secretaria de Vigilância a Saúde [document on the internet]. Brasília (DF): Ministério da Saúde; 2014 [cited 2018 May 17]. Available from: http://portalms.saude.gov. $\underline{\mathrm{br} / \mathrm{svs}}$

6. Nettina SM. Prática de enfermagem. Rio de Janeiro: Guanabara Koogan; 2012.

7. Rodrigues JD, Cruz MS, Paixão AN. Uma análise da prevenção do câncer de mama no Brasil. Ciênc Saúde Colet [serial on the internet]. 2015 [cited 2018 May 17];20(10):3163-76. Available from: http://www.scielo.br/ $\mathrm{pdf} / \mathrm{csc} / \mathrm{v} 20 \mathrm{n} 10 / 1413-8123-\mathrm{csc}-20-10-3163 . \mathrm{pdf}$

8. World Health Organization. International Agency for Research on Cancer. Geneva: WH0; 2016.

9. Brasil. Estimativa 2016/2017. Estimativa por tipo de câncer. Rio de Janeiro: Instituto Nacional de Câncer; 2016.

10. Brasil. Doenças crônicas: câncer. Brasília (DF): Ministério da Saúde; 2014.

11. Arruda RL, Teles ED, Oliveira FJF, Fontoura IG, Ferreira AGN, Machado NS. Prevenção do câncer de mama em mulheres atendidas em unidades básicas de saúde. Rev Rene [serial on the internet]. 2015 [cited 2017 Jan 20];16(2):143-9. Available from: http://www.repositorio.ufc.br/bitstream/ riufc/12638/1/2015_art_rlarruda.pdf
12. Brouselle A, Champagne F, Contandriopoulos AP, Hartz $Z$, organizers. Avaliação: conceitos e métodos. Rio de Janeiro: Ed. Fiocruz; 2011.

13. Botelho LLR, Cunha CCA, Macedo M. 0 método da revisão integrativa nos estudos organizacionais. Gestão Sociedade [serial on the internet]. 2011 [cited 2018 May 17];5(11):12136. Available from: https://www.gestaoesociedade.org/ gestaoesociedade/article/view/1220/906

14. Cavalcanti LPG, Simões PSF, Silva MRR, Galdino PNR. Assistência em mastologia em uma unidade de referência do Sistema Único de Saúde no Ceará, Brasil. Rev Bras Cancerol. 2012;58(4):603-9. Available from: http://www.inca.gov.br/ $\mathrm{rbc} / \mathrm{n} 58 / \mathrm{v} 04 / \mathrm{pdf} / 05$-artigo-assistencia-mastologiaunidade-referencia-sistema-unico-saude-ceara-brasil.pdf

15. Barros AF, Uemura G, Macedo JLS. Atraso no diagnóstico e tratamento do câncer de mama e estratégias para sua redução. Femina. 2012;40(1):31-6.

16. Lourenço TS, Mauad EC, Vieira AC. Barreiras no rastreamento do câncer de mama e o papel da enfermagem: revisão integrativa. Rev Bras Enferm [serial on the internet]. 2013 [cited 2018 May 17];66(4):585-91. Available from: http://www.scielo.br/pdf/reben/v66n4/v66n4a18.pdf

17. Nicolaou PK, Padoin LV. 0 retrato das políticas públicas no tratamento do câncer de mama no Brasil. Rev Bras Mastologia [serial on the internet]. 2013 [cited 2018 May 17];23(3):92-4. Available from: http://www.rbmastologia. com.br/wp-content/uploads/2015/06/MAS_v23n3_92-94. $\underline{\mathrm{pdf}}$

18. Bertocchi FM, Fernandes BM, Almeida MIG, Freitas SC, Paiva CCN, Paula EA. Conduta de profissionais durante a consulta de rastreio do câncer de mama e útero. Rev Rene [serial on the internet]. 2014 [cited $2017 \mathrm{Jan} 20$ ];15(6):9739. Available from: http://www.revistarene.ufc.br/revista/ index.php/revista/article/viewFile/1803/pdf

19. Azevedo e Silva G, Bustamante-Teixeira MT, Aquino EML, Tomazelli JG, Santos SI. Acesso à detecção precoce do câncer de mama no Sistema Único de Saúde: uma análise a partir dos dados do Sistema de Informação em Saúde. Cad Saúde Pública [serial on the internet]. 2014 [cited 2018 May 17];30(7):1537-50. Available from: http://www.scielo. $\mathrm{br} / \mathrm{pdf} / \mathrm{csp} / \mathrm{v30n7/0102-311X-csp-30-7-1537.pdf}$

20. Paiva CJK, Cesse EAP. Aspectos relacionados ao atraso no diagnóstico e tratamento do câncer de mama em uma unidade hospitalar de Pernambuco. Rev Bras Cancerol [serial on the internet]. 2015 [cited 2018 May 17];61(1):23-30. Available from: http://www.inca.gov.br/rbc/n_61/v01/ pdf/05-artigo-aspectos-relacionados-ao-atraso-nodiagnostico-e-tratamento-do-cancer-de-mama-em-umaunidade-hospitalar-de-pernambuco.pdf

21. Feijó AM, Leon LC, Costa VA, Pozza SB. Os caminhos de cuidado das mulheres com diagnóstico de câncer de mama. Av Enferm [serial on the internet]. 2016 [cited 2018 May 17];34(1):58-68. Available from: http://www.scielo.org.co/ $\mathrm{pdf} / \mathrm{aven} / \mathrm{v} 34 \mathrm{n} 1 / \mathrm{v} 34 \mathrm{n} 1 \mathrm{a} 07 . \mathrm{pdf}$ 
22. Traldi MC, Galvão P, Morais SS, Fonseca MRCC. Demora no diagnóstico de câncer de mama de mulheres atendidas no sistema público de saúde. Cad Saúde Coletiva [serial on the internet]. 2016 [cited 2018 May 17];24(2):185-91. Available from: http://www.scielo.br/pdf/cadsc/v24n2/1414-462Xcadsc-24-2-185.pdf

23. Brasil. Controle do câncer de mama. Rio de Janeiro: Instituto Nacional de Câncer; 2016.

24. Brasil. Lei n. 12.732, de 22 de novembro de 2012. Dispõe sobre o primeiro tratamento de paciente com neoplasia maligna comprovada e estabelece prazo para seu início. Diário Oficial da União [serial on the internet], Brasília (2012 Nov 23 [cited 2018 May 17]); Sec 1. Available from: http://www.planalto.gov.br/ccivil_03/_ato20112014/2012/lei/l12732.htm

25. Brasil. Diretrizes para a detecção precoce do câncer de mama no Brasil. Rio de Janeiro: Instituto Nacional de Câncer; 2015.

26. Farias LMA, Aguiar VCF, Carvalho AMF, Linhares JM, Linhares AEMS, Sousa AMM. Grupo de mulheres mastectomizadas: construindo estratégias de cuidado. Sanare (Sobral, 0nline) [serial on the internet]. 2014 [cited 2018 May 17];14(2):91-7. Available from: https://sanare. emnuvens.com.br/sanare/article/view/831/502

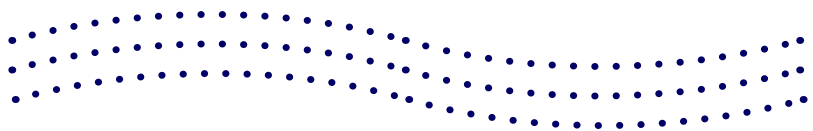
$\ldots . .$.

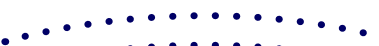

$\ldots \ldots \ldots \ldots \ldots \ldots$ $\ldots \ldots \ldots \ldots \ldots \ldots \ldots \ldots$ $\ldots \ldots \ldots \ldots \ldots \ldots$ 西, 